\title{
BMJ Open Complementary therapies for labour and birth study: a randomised controlled trial of antenatal integrative medicine for pain management in labour
}

\author{
Kate M Levett, ${ }^{1} \mathrm{C}$ A Smith, ${ }^{1}$ A Bensoussan, ${ }^{1} \mathrm{H}$ G Dahlen ${ }^{2}$
}

To cite: Levett KM, Smith CA Bensoussan A, et al. Complementary therapies for labour and birth study: a randomised controlled trial of antenatal integrative medicine for pain management in labour. BMJ Open 2016;6: e010691. doi:10.1136/ bmjopen-2015-010691

- Prepublication history and additional material is available. To view please visit the journal (http://dx.doi.org/ 10.1136/bmjopen-2015010691).

Received 27 November 2015 Revised 30 April 2016 Accepted 3 May 2016

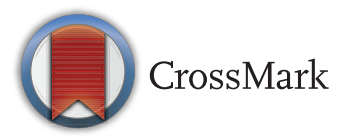

\footnotetext{
${ }^{1}$ National Institute for Complementary Medicines (NICM), Western Sydney University, Sydney, Australia ${ }^{2}$ School of Nursing and Midwifery, Western Sydney University, Sydney, Australia

Correspondence to Dr Kate M Levett; K.Levett@ westernsydney.edu.au
}

\section{ABSTRACT}

Objective: To evaluate the effect of an antenatal integrative medicine education programme in addition to usual care for nulliparous women on intrapartum epidural use.

Design: Open-label, assessor blind, randomised controlled trial.

Setting: 2 public hospitals in Sydney, Australia. Population: 176 nulliparous women with low-risk pregnancies, attending hospital-based antenatal clinics.

Methods and intervention: The Complementary Therapies for Labour and Birth protocol, based on the She Births and acupressure for labour and birth courses, incorporated 6 evidence-based complementary medicine techniques: acupressure, visualisation and relaxation, breathing, massage, yoga techniques, and facilitated partner support. Randomisation occurred at 24-36 weeks' gestation, and participants attended a 2-day antenatal education programme plus standard care, or standard care alone. Main outcome measures: Rate of analgesic epidural use. Secondary: onset of labour, augmentation, mode of birth, newborn outcomes.

Results: There was a significant difference in epidural use between the 2 groups: study group $(23.9 \%)$ standard care $(68.7 \%$; risk ratio (RR) $0.37(95 \% \mathrm{Cl}$ 0.25 to 0.55$), p \leq 0.001)$. The study group participants reported a reduced rate of augmentation ( $R R=0.54$ $(95 \% \mathrm{Cl} 0.38$ to 0.77$), p<0.0001)$; caesarean section ( $R R=0.52(95 \% \mathrm{Cl} 0.31$ to 0.87$), p=0.017)$; length of second stage (mean difference $=-0.32(95 \% \mathrm{Cl}-0.64$ to 0.002$), p=0.05)$; any perineal trauma $(0.88(95 \% \mathrm{Cl}$ 0.78 to 0.98$), p=0.02$ ) and resuscitation of the newborn ( $R R=0.47$ ( $95 \% \mathrm{Cl} 0.25$ to 0.87 ), $\mathrm{p} \leq 0.015$ ). There were no statistically significant differences found in spontaneous onset of labour, pethidine use, rate of postpartum haemorrhage, major perineal trauma (third and fourth degree tears/episiotomy), or admission to special care nursery/neonatal intensive care unit $(p=0.25)$.

Conclusions: The Complementary Therapies for Labour and Birth study protocol significantly reduced epidural use and caesarean section. This study provides evidence for integrative medicine as an

\section{Strengths and limitations of this study}

- This is the first randomised controlled trial in Australia that has investigated the effectiveness of a birth preparation course, integrating multiple complementary medicine (CM) techniques, for the support of natural birth for first-time mothers. This suggests a reorientation of antenatal education towards normal birth, and reflects current outcome measures in reports of maternity services policy directives.

- The study used self-administered, evidencebased $\mathrm{CM}$ techniques and blinded analysis to test an a priori hypothesis, and implemented a pragmatic design where participants were free to use any of the techniques with no prescriptions or time limitations for use, allowing women and partners to have control and influence over their birth process, and use information and $\mathrm{CM}$ tools to manage their own labours.

- The primary outcome measure of epidural block (EDB) was used, rather than frequently used pain scores, as the objective measure of EDB has been identified as a mediating factor in labour interventions and mode of birth, described as the cascade of interventions.

- Limitations of this study include higher enrolment of relatively wealthy, well-educated women and relatively fewer participants from the area identified as lower socioeconomic status. This is in line with previous CM research, but it is worth considering that the highest rates of epidural use and caesarean section are also among this more advantaged population.

- Wider national and international implementation of this study is recommended to confirm results in a broader population and examine issues of generalisability.

effective adjunct to antenatal education, and contributes to the body of best practice evidence.

Trial registration number: ACTRN12611001126909. 


\section{INTRODUCTION}

There has been a rise in rates of intervention during labour and birth in most developed countries, ${ }^{1}{ }^{2}$ and the intervention rates in Australia during birth are well above the Organisation for Economic Cooperation and Development (OECD) averages. ${ }^{3}$ As these interventions increase, such as routine use of epidural block (EDB), so does the rate of instrumental births and associated medical interventions. ${ }^{4-8}$ Epidural rates in New South Wales (NSW) hospitals have shown a rapid rise over the past decade. In 2012, the state average for EDB use was $46.5 \%$; however, there was broad variation within the state, ranging from $15 \%$ to $82.7 \%$ depending on region and hospital. ${ }^{9} 10$ The high use of EDB for pain relief in labour has been identified as a contributing factor in rising rates of augmentation and assisted vaginal births and caesarean section (CS). ${ }^{4-8} 11$

Childbirth education has also seen a shift away from birth preparation ${ }^{12} 13$ to a curriculum broadly centred on overall parent education. ${ }^{14}$ Findings from a systematic review on childbirth education reports that the effectiveness of antenatal education for childbirth or parenthood supports the idea that educational interventions have a role in increasing feelings of self-confidence and control, but demonstrates little impact on reducing interventions and associated morbidity in labour. ${ }^{15} 16$

Integrative medicine approaches and complementary medicine (CM), in particular, may offer increased options for pain relief in birth, ${ }^{17}$ and may be effective within the hospital antenatal education framework. The term integrative medicine is used when referring to incorporating $\mathrm{CM}$ or complementary therapies (CT) into mainstream healthcare. ${ }^{18}$ Recent Australian data suggest that $74.4 \%$ of women used some form of CM during pregnancy, and $66.7 \%$ of these women also used non-pharmacological pain relief in labour. ${ }^{19}$ The Cochrane Systematic Review on pain management for labour finds some evidence to suggest that acupuncture, relaxation, massage and water immersion may assist in the management of labour with few side effects; however, more research is needed to establish efficacy of these techniques. ${ }^{20}$

In response to the need to establish the evidence base for CM interventions for pain management in labour, we undertook a randomised controlled trial (RCT) to test the hypothesis that nulliparous women who undergo a CM antenatal education course, in addition to usual antenatal care, would use less EDB than nulliparous women who receive usual antenatal care alone. Trials of complex interventions are difficult to conduct and do not have linear models, and require a pragmatic approach to implementation. ${ }^{21} 22$

\section{METHODS}

The Complementary Therapies for Labour and Birth (CTLB) study protocol was based on the She Births Antenatal Education Program, with an acupressure component 'acupressure techniques for use during childbirth and pregnancy' protocol. ${ }^{23}$ The study protocol was further adapted to reflect the evidence base for the CM techniques that were incorporated. $.^{22} 24-26$

From April 2012 to August 2013, women and their birth partners were recruited to a two-arm study consisting of a study group, who received the CTLB protocol in addition to usual care, and a control group, who received usual care alone. The study was an assessorblinded, open-label pragmatic RCT.

\section{Participants}

Women attending antenatal clinic were eligible to participate in the study from 24 to 34 weeks' gestation. They were provided with a participant information sheet for themselves and their birth partner. If they were interested and eligible to participate, women and partners signed individual consent forms. Women were eligible to enter the trial if they had a singleton pregnancy with a cephalic presentation, were low risk (no pre-existing medical complications or existing obstetric complications), were first-time mothers (nulliparous) and had knowledge of sufficient English to participate in a course. Women were excluded from entering the trial if they had preidentified risk factors, were enrolled or intending to enrol in a 'continuity of care' midwifery programme or in a private birth preparation course, were unable to attend a weekend course, knew insufficient English for participation, or had been previously randomised to the trial. Recruitment was undertaken at two public hospitals in Sydney, Australia, that reflected diverse socioeconomic areas. Recruitment was also conducted through the affiliated Western Sydney University (WSU) in response to newspaper and magazine advertisements. Participants who were recruited through WSU attended the courses at either of the two hospital study sites. All eligible women were approached in the antenatal clinic at site 1 as this was a smaller unit and individual contact was possible; all clinics were attended regularly by the researcher. At site 2, the hospital was much larger and more diverse with regard to structure of the clinics. Different clinics were attended, and eligible women at these clinics were approached. It was not possible to attend all clinics at this larger unit, and a range of clinics were selected on different weeks to achieve a representative sample of women. For site 3, where flyers and newspaper advertisements were used for recruitment, the response rate was quite low. All eligible women who contacted us through these means were randomised to the study. We do not have data on the women who were not eligible to participate. The randomisation target was achieved quite quickly, and participation was popular.

\section{Randomisation}

We used a web-based computer-generated randomisation sequence prepared centrally via the 'Sealed Envelope' website (https://http://www.sealedenvelope.com), and 
concealed centrally. Stratification occurred for hospital site, yielding three randomisation lists: 'site $\mathrm{H}$ ', 'site N' and 'WSU'. Women were randomly allocated to either the study group or usual care alone. Randomisation occurred on a 1:1 allocation ratio to ensure equal numbers in each group at each hospital. All randomisations were entered by the investigator KL.

\section{Intervention}

Two-day courses (see online supplementary file 1) were conducted over a weekend at one of the two hospital venues on a fortnightly to monthly basis over a 15-month period from May 2012 to August 2013. A total of 20 courses were conducted during this time. Participants attended prior to 36 weeks' gestation with a birth partner, and there was a maximum of 12 couples and a minimum of 2 couples at each course, with an average of 8 couples per course. The study investigator (KL) ran each course.

The underlying philosophy and specific techniques included in the intervention programme were designed to support a woman during her pregnancy and labour by introducing tools to enhance a natural state of relaxation (visualisation, breathing, massage, yoga), and facilitate labour progression (yoga, acupressure) and pain relief (breathing, acupressure, visualisation). The CTLB protocol introduces concepts of birth as a natural physiological process, and the idea of "working with pain",27 using evidence-based CM tools by which the birth process can be managed. ${ }^{20}$ Women and partners received education about the physiology of normal birth.

The tools used were:

1. Visualisation ${ }^{25}$-four guided visualisations rehearsed through the courses and given to participants on a CD to practice at home;

2. Yoga postures ${ }^{28}$-five postures and movements practiced to encourage relaxation, physiological position for labour, opening of the pelvis and downward descent of the baby;

3. Breathing techniques ${ }^{20}$-four breathing techniques were introduced: soft sleep breaths for relaxation between contractions; blissful belly breaths (BBs) which were used during contractions for pain relief; Cleansing Calming Breaths used following contractions during the transition period of labour; and the gentle birthing breath (GB) which was for use during the second stage of labour and encouraged descent of the baby avoiding active pushing and protection of the pelvic floor;

4. Massage ${ }^{26}$ - two techniques were shown to partners: the endorphin massage used between contractions, which is a soft technique and encourages endorphin release; and the stronger massage which is used during contractions for pain relief and focuses on squeezing the buttock, especially the piriformis muscle, to interrupt pain perception;

5. Acupressure, ${ }^{22} 24$ which uses six main points for use during labour selected from a previously published protocol. ${ }^{23}$ These focus on hormone release for labour progression, augmentation of contractions, pain relief, nausea and positioning of baby;

6. Facilitated partner support ${ }^{29-31}$ uses the concept of working with pain ${ }^{27}$ and instructs partners to advocate for the labouring woman, promoting her oxytocin levels and minimising her stress with actions and techniques which are supportive for the birthing woman, and gives time for facilitated discussion and rehearsal by couples during the course.

Usual care consisted of the hospital-based antenatal education course routinely available at each hospital. Antenatal education classes in Australia currently take a general descriptive approach to labour preparation, and emphasise parenting and postpartum issues as the main focus. ${ }^{14}$ Classes generally run weekly over $6-7$ weeks or over 1-2 weekends, and include topics such as: pregnancy changes, exercise and back care during pregnancy, signs of labour, unexpected outcomes in labour and birth, pharmacological pain management, managing labour and birth, newborn care and breast feeding, parenthood, and baby's first weeks.

\section{Blinding}

Women, their partners and the chief investigator (KL) were not blinded to group allocation. Group allocated was subsequently coded, and outcome measures were assessed and analysed blind to study group allocation. Midwives and doctors at each of the two main study hospitals and other sites were aware of the study, but delivery suite personnel were blinded to study participants' group allocation. Study course content was not disclosed to midwives to avoid any change in practice that may occur. Group allocation and data were linked by identification codes, allowing for the analysis to be undertaken blind.

\section{A priori outcome measures}

Primary outcome: epidural use for pain relief.

Secondary outcomes: other pharmacological pain relief use during labour; induction of labour; augmentation of labour; length of labour; instrumental delivery; CS, postpartum haemorrhage (PPH; >500 mL blood loss); perineal trauma (first/second/third/fourth degree tear/ episiotomy); major or severe perineal trauma (third/ fourth degree tear/episiotomy); Apgar scores $<7$ at $5 \mathrm{~min}$; resuscitation of the newborn (with oxygen, suction, bag and mask, intubation, cardiac massage); admission to special care nursery/neonatal intensive care unit (SCN/NICU). Other outcomes included attitude towards birth and personal sense of control, as well as postnatal depression at 6 weeks, measured by the Edinburgh Postnatal Depression Scale (EPDS). ${ }^{32}$

\section{Questionnaires}

To assess measures of personal control, we used the Labour Agentry Scale (LAS).$^{33}$ Within 72 hours following birth the LAS was administered to all women (see online supplementary file 2). The LAS contains 29 
questions with a seven-point Likert scale ranging from ' $1=$ almost always', to ' 7 =rarely'. Therefore, scores could theoretically range from 29, indicating the highest control possible, to a high score of 203 indicating the lowest agency possible. Clinical outcomes were collected from hospital birth records and the NSW Hospitals' birth summaries, which were accessed from the hospitals where the participant had given birth.

\section{Analysis}

An intention-to-treat (ITT) analysis was used for the primary and secondary outcome data. The $\chi^{2}$ and t-tests were used for univariate analysis of categorical and continuous data, respectively. Significance was set at an $\alpha$ of 0.05 , reporting on relative risk with a $95 \%$ CI. Group allocation was coded by an independent researcher, and the investigator undertook the analysis blind to group allocation. Data were analysed using SPSS V.22 (IBM SPSS Statistics for Windows, Version 22.0 [program]. Armonk, KY: IBM Corp, 2013).

\section{Sample size and power}

The trial was designed to demonstrate an absolute reduction of $20 \%$ in epidural use from $46 \%$ in those women managed with usual care to $26 \%$ in those women who were randomised to the study. The rate of use of EDB was determined by published data for the two study hospitals in the 2011 NSW Mothers and Babies Report. ${ }^{34}$ This required a total sample size of 170 women for $80 \%$ power at a significance level of $\mathrm{p}<0.05$. Recruitment continued until at least 170 women had been enrolled, and those randomised to the treatment group had either completed the course or were known to have missed their course, with 176 randomised and 171 completing the study. A low drop-out rate $(<3 \%)$ was observed for the overall study population, and separately for each arm of the study $(<5 \%) .{ }^{35}$ Primary outcome data were available for all consenting participants.

\section{RESULTS}

We assessed 315 women for eligibility to participate in the study, of whom, 176 were randomised and 171 were included in the final analysis (figure 1). Five women were lost to follow-up. Women were randomised to the study group $(n=89)$ or the control group $(n=87)$. From the 315 women screened, 139 were excluded for the following reasons: 105 declined to participate, and 34 did not meet inclusion criteria (insufficient English $(n=7)$, attending private birth preparation course $(n=12)$,

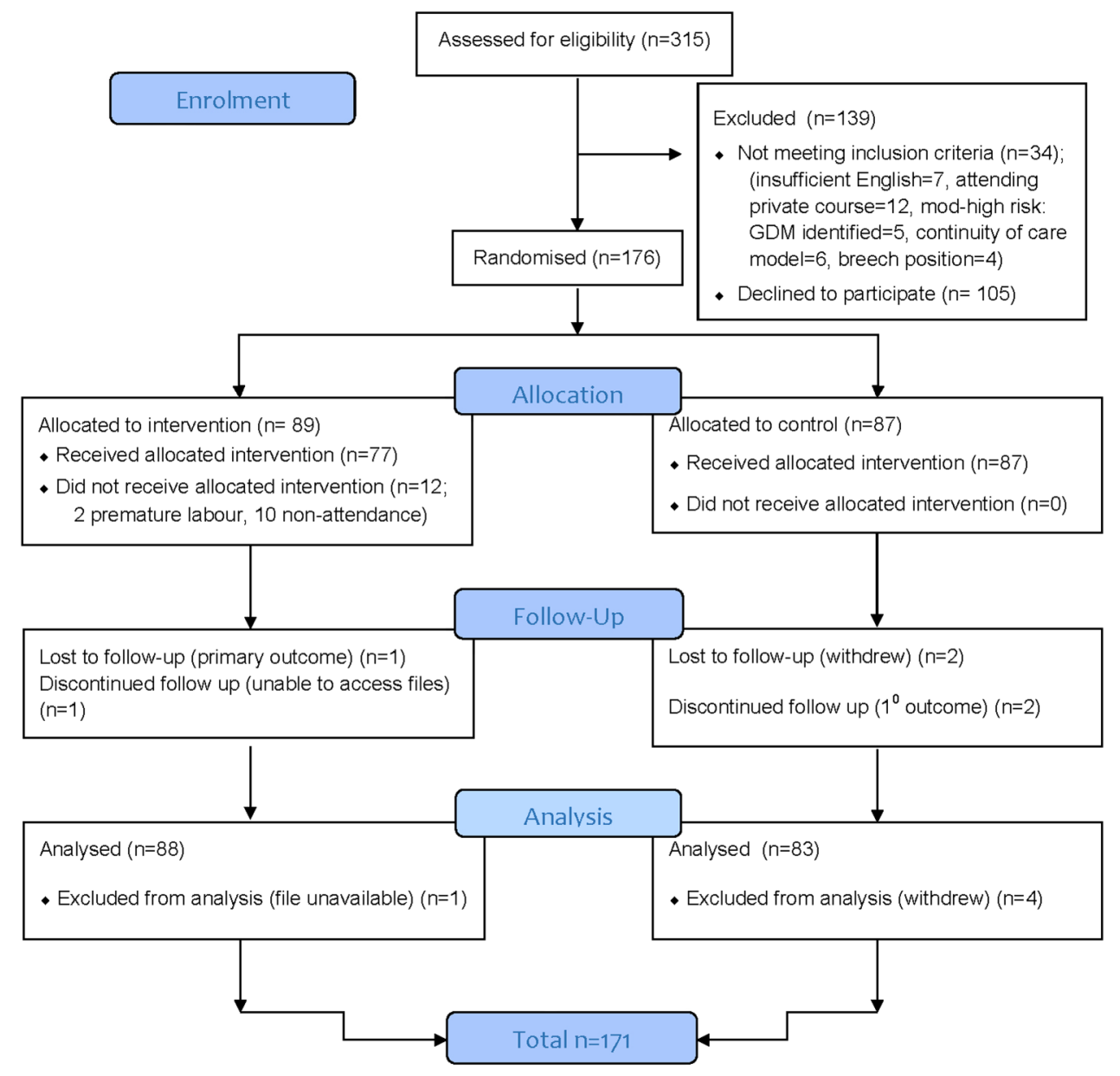

Figure 1 CONSORT flow chart of CTLB study. 
continuity of care model $(\mathrm{n}=6)$, moderate-to-high risk gestational diabetes mellitus (GDM) $(n=5)$, breech presentation $(n=4))$. In the final analysis, there were 101 women included from site 1, 30 women from site 2 and 40 women from site 3 .

All women completed the trial entry form at baseline, including the demographic information. Not all women answered each question in the trial entry form (table 1). Following birth, the LAS was completed by 72 of the 88 women (82\%) in the study group, and 52 of the 83 (62\%) women in the control group.

Participants in the intervention group did not significantly differ from those in the control group in terms of their age, body mass index, cultural background, level of education, income, hospital status or model of care (table 1). Babies were not different in terms of average gestational age or weight at birth.

\section{Primary outcome}

A statistically and clinically significant reduction in epidural rate was found for the intervention group compared with the control group. The overall unadjusted rate of EDB in the control group was $68.7 \%$, and $23.9 \%$ in the study group (risk ratio $(\mathrm{RR})=0.35$ (0.23 to 0.52 ), $\mathrm{p} \leq 0.0001$; table 2 ). In addition to stratification of randomisation by site, a post hoc analysis was performed for each site. The RRs were similar to the primary analysis (RR1=0.27 (0.12 to 0.60), RR2=0.31 (0.11 to 0.90),

\begin{tabular}{|c|c|c|}
\hline $\begin{array}{l}\text { Demographic } \\
\text { characteristics }\end{array}$ & $\begin{array}{l}\text { Study group, } \\
\mathrm{n}=87\end{array}$ & $\begin{array}{l}\text { Control group, } \\
\mathrm{n}=85\end{array}$ \\
\hline Mean age (years, \pm SD) & $30.41( \pm 4.99)$ & $28.87( \pm 5.24)$ \\
\hline $\mathrm{BMI}($ mean \pm SD) & $22.66( \pm 4.47)$ & $23.35( \pm 3.93)$ \\
\hline Cultural background & $\mathrm{n}=79(\%)$ & $\mathrm{n}=61(\%)$ \\
\hline Caucasian & $58(73.4)$ & $44(72.1)$ \\
\hline Asian & $10(12.7)$ & $11(18.0)$ \\
\hline Other & $11(13.9)$ & $6(9.9)$ \\
\hline Income & $\mathrm{n}=78(\%)$ & $\mathrm{n}=61(\%)$ \\
\hline$<60$ & $12(15.4)$ & $12(19.7)$ \\
\hline $60-80 \mathrm{k}$ & $7(9.0)$ & $10(16.4)$ \\
\hline $80-100 k$ & $17(21.8)$ & $10(16.4)$ \\
\hline$>100 \mathrm{k}$ & $42(53.5)$ & $29(47.5)$ \\
\hline Total & 78 & 61 \\
\hline Education & $\mathrm{n}=81(\%)$ & $\mathrm{n}=60(\%)$ \\
\hline $\begin{array}{l}\text { High school/ } \\
\text { vocational }\end{array}$ & $24(29.6)$ & $20(33.3)$ \\
\hline $\begin{array}{l}\text { University/ } \\
\text { postgraduate }\end{array}$ & $57(70.4)$ & $40(66.7)$ \\
\hline Hospital status & $\mathrm{n}=87(\%)$ & $\mathrm{n}=85(\%)$ \\
\hline Public status & $82(94.3)$ & 79 (92.9) \\
\hline Private status & $5(5.7)$ & $6(7.1)$ \\
\hline Model of care & $\mathrm{n}=87(\%)$ & $\mathrm{n}=85(\%)$ \\
\hline Midwifery & 67 (82.7) & 64 (85.3) \\
\hline Doctors care & $4(4.9)$ & $7(9.3)$ \\
\hline Shared care & $10(12.3)$ & $4(5.3)$ \\
\hline
\end{tabular}

RR3=0.39 (0.23 to 0.65$))$. Using a true ITT analysis, we examined the data, including data points for the five women who had dropped out, withdrawn or were lost to follow-up. There were four in the control group, and one in the study group. Using a best-case-worst-case scenario, we included the five cases with missing data for the primary outcome. If the four control group women did not have an EDB and the one study group woman did have an EDB (worst case), the results were still highly statistically significant with a RR of $0.40(95 \%$ CI 0.27 to 0.59$), \mathrm{p}=<0.0001$.

\section{Secondary clinical outcomes}

Women in the study group were more likely to experience a normal vaginal birth (NVB) $(\mathrm{RR}=1.56$ (1.12 to $2.17), \mathrm{p}=<0.01$ ), and were less likely to have medical or surgical augmentation during labour $(\mathrm{RR}=0.54(95 \% \mathrm{CI}$ 0.38 to 0.77$), \mathrm{p}<0.001)$, birth by $\mathrm{CS}(\mathrm{RR}=0.52(0.31$ to $0.87), \mathrm{p}=0.01)$ or any perineal trauma $(\mathrm{RR}=0.88(95 \%$ CI 0.7 to 0.98$), p=0.02$ ). We also found a reduced length of second stage of labour (mean difference $(\mathrm{MD})=-0.32$ (95\% CI -0.64 to 0.002$), \mathrm{p}=0.05$ ) in the study group (table 2). Babies of women in the study group were also less likely to require resuscitation by suction ( \pm oxygen) or with bag and mask ( $\mathrm{RR}=0.47$ (95\% CI 0.25 to 0.87 ), $\mathrm{p}=0.015$ ). There were no differences in the rare outcomes of intubation or cardiac massage required at birth. Only one baby in the study group required intubation. There were some non-significant trends towards the study group having less likelihood of an instrumental vaginal birth (VB) ( $\mathrm{RR}=0.57 \quad(95 \%$ CI 0.30 to 1.09$)$, $\mathrm{p}=0.09$ ), and nitrous oxide (gas) for pain management $(\mathrm{RR}=0.77$ (95\% CI 0.57 to 1.03 ), $\mathrm{p}=0.09$ ).

No significant differences were found in the secondary outcome measures of spontaneous onset of labour ( $\mathrm{RR}=1.13(95 \%$ CI 0.82 to 1.57$), \mathrm{p}=0.51)$, pethidine use $(\mathrm{RR}=1.11$ (95\% CI 0.65 to 2.2), $\mathrm{p}=0.56)$, rates of $\mathrm{PPH}$ $(\mathrm{RR}=0.82(95 \%$ CI 0.41 to 1.61$), \mathrm{p}=0.85)$ or major perineal trauma (third/fourth degree tear or episiotomy; $\mathrm{RR}=0.94$ (95\% CI 0.57 to 1.55 ), $\mathrm{p}=0.85$ ). No significant differences were found in Apgar scores $(\mathrm{RR}=0.99(95 \%$ CI 0.95 to 1.03 ), $\mathrm{p}=1.0$ ), or admission to the SCN/NICU $(\mathrm{RR}=0.59$ (95\% CI 0.24 to 1.46$), \mathrm{p}=0.25)$.

The length of the second stage of labour was 1 hour for the study group and1 hour $32 \mathrm{~min}$ for the control group, resulting in a MD of $32 \mathrm{~min}(\mathrm{p}=0.05)$. There were no significant differences between the groups for the first stage of labour or the total length of labour (see table 3).

The LAS questionnaire examined whether the course had any impact on attitudes and feelings about birth and women's feelings of agentry. The LAS was completed by 72 of the 88 women in the study group (82\%), with an average score of $164.97 \quad(\mathrm{SD}=27.06)$. In the control group 52 of the 83 women (62\%) completed the form, and had an average score of $150.92(\mathrm{SD}=30.03)$. We found a statistically significant difference between the two groups for this score ( $\mathrm{MD}=14.05,95 \%$ CI 3.84 to 24.26, $\mathrm{p}<0.01)$. 
Table 2 Unadjusted primary and secondary outcomes measures

\begin{tabular}{|c|c|c|c|}
\hline Outcomes & $\begin{array}{l}\text { Study group } \\
(n=88) \%\end{array}$ & $\begin{array}{l}\text { Control group } \\
(n=83) \%\end{array}$ & Risk ratio \\
\hline Epidural analgesia & $21(23.9)$ & $57(68.7)$ & $\begin{array}{l}0.35(0.23 \text { to } 0.52) \\
p<0.0001^{\star *}\end{array}$ \\
\hline Spontaneous onset labour & $62(70.5)$ & $54(65.1)$ & $\begin{array}{l}1.13(0.82 \text { to } 1.57) \\
p=0.51\end{array}$ \\
\hline Augmentation & $25(28.4)$ & $48(57.8)$ & $\begin{array}{l}0.54(0.38 \text { to } 0.77) \\
p<0.0001^{\star *}\end{array}$ \\
\hline Mode of birth: NVB & $60(68.2)$ & $39(47.0)$ & $\begin{array}{l}1.56(1.12 \text { to } 2.17) \\
p \leq 0.01^{\star *}\end{array}$ \\
\hline Mode of birth: CS & $16(18.2)$ & $27(32.5)$ & $\begin{array}{l}0.52(0.31 \text { to } 0.87) \\
p=0.017^{\star}\end{array}$ \\
\hline Mode of birth: instrumental & $12(13.6)$ & $17(20.5)$ & $\begin{array}{l}0.57(0.30 \text { to } 1.09) \\
p=0.09\end{array}$ \\
\hline Nitrous oxide (gas) & $40(45.5)$ & $49(59.0)$ & $\begin{array}{l}0.77(0.57 \text { to } 1.03) \\
p=0.092\end{array}$ \\
\hline Pethidine & $19(20.5)$ & $15(19.3)$ & $\begin{array}{l}1.11(0.78 \text { to } 1.56) \\
p=0.70\end{array}$ \\
\hline $\begin{array}{l}\text { Any perineal trauma } \\
\dagger(\text { trauma/VB) }\end{array}$ & $61 / 72(84.7) \dagger$ & $54 / 56(96.4) \dagger$ & $\begin{array}{l}0.88(0.78 \text { to } 0.98) \\
p=0.02^{*}\end{array}$ \\
\hline $\begin{array}{l}\text { Major perineal trauma } \\
\text { †(trauma/VB) }\end{array}$ & 49/72 (68.1)† & $37 / 56(66.1) \dagger$ & $\begin{array}{l}0.94(0.57 \text { to } 1.55) \\
p=0.85\end{array}$ \\
\hline $\mathrm{PPH}$ & $13(14.8)$ & $15(18.1)$ & $\begin{array}{l}0.82(0.41 \text { to } 1.61) \\
p=0.68\end{array}$ \\
\hline Resuscitation (suction $\pm \mathrm{O}_{2} /$ bag and mask) & $12(13.6)$ & $24(28.9)$ & $\begin{array}{l}0.47(0.25 \text { to } 0.87) \\
p=0.015^{*}\end{array}$ \\
\hline Apgar <7 (5 min) & $3(3.4)$ & $4(4.8)$ & $\begin{array}{l}0.99(0.95 \text { to } 1.03) \\
p=1.0\end{array}$ \\
\hline NICU/SCN admit & $7(8.0)$ & $11(13.2)$ & $\begin{array}{l}0.59(0.24 \text { to } 1.46) \\
p=0.25\end{array}$ \\
\hline
\end{tabular}

${ }^{*}<0.05 ;{ }^{* *}<0.01$.

†Percentage is from all vaginal births: denominator $=72$ in study group and 56 in control group. Major perineal trauma is defined as third or fourth degree tear and episiotomy.

$\mathrm{CS}$, caesarean section; NICU, neonatal intensive care unit; PPH, postpartum haemorrhage; SCN, special care nursery.

Given that a large number of women did not complete this form, there is the possibility of reporting bias in the results. We used a Levene's test for equality of variance, and found the variance between the two groups was not significantly different $(p=0.59)$. Additionally, we did a post hoc analysis to determine if any differences were present between the study group and the control group for baseline characteristics by controlling for responders versus non-responders. No differences were found between groups.
Six weeks following the birth, participants completed an EPDS questionnaire. However, there was a high rate of non-compliance with this form: 27 women in the study group and 41 women in the control group did not complete this form. There was no statistically significant difference between groups at 6-week follow-up for this cohort of women (see table 4).

Analysis of patterns of CM use in labour reveal women in the study group used an average of $3.94 \quad(\mathrm{SD}=1.4)$ techniques during labour, and in the antenatal period

Table 3 Length of labour

\begin{tabular}{|c|c|c|c|}
\hline $\begin{array}{l}\text { Outcomes } \\
\text { Length of labour }\end{array}$ & $\begin{array}{l}\text { Study group }(n=86) \\
\text { Mean (SD) }\end{array}$ & $\begin{array}{l}\text { Control group }(n=83) \\
\text { Mean (SD) }\end{array}$ & $\begin{array}{l}\text { Difference statistic } \\
\text { MD }(95 \% \mathrm{Cl}) \\
\text { p Value }\end{array}$ \\
\hline First stage & $6.12(3.95)$ & $6.53(3.90)$ & $\begin{array}{l}M D=-0.41(-1.79 \text { to } 0.98) \\
p=0.56\end{array}$ \\
\hline Second stage & $1.00(0.87)$ & $1.32(0.98)$ & $\begin{array}{l}\mathrm{MD}=-0.32(-0.64 \text { to } 0.002) \\
\mathrm{p}=0.05^{\star}\end{array}$ \\
\hline Total length of labour & $7.43(4.13)$ & $8.20(4.37)$ & $\begin{array}{l}\mathrm{MD}=-0.77(-2.26 \text { to } 0.72) \\
\mathrm{p}=0.31\end{array}$ \\
\hline
\end{tabular}


Table 4 Six-week postpartum: EPDS

\begin{tabular}{|c|c|c|c|}
\hline $\begin{array}{l}\text { EDPS } \\
\text { Postnatal }\end{array}$ & $\begin{array}{l}\text { Study } \\
\text { group } \\
\mathrm{n}=61 \\
\text { Mean (SD) } \\
\end{array}$ & $\begin{array}{l}\text { Control } \\
\text { group } \\
n=42 \\
\text { Mean (SD) }\end{array}$ & $\begin{array}{l}\text { Mean } \\
\text { difference } \\
95 \% \mathrm{Cl} \\
\text { p Value } \\
\end{array}$ \\
\hline $\begin{array}{l}\text { Postnatal } \\
\text { EPDS }\end{array}$ & $4.49(3.44)$ & 4.07 (3.93) & $\begin{array}{l}\mathrm{MD}=0.42 \\
(-1.03 \text { to } 1.87) \\
\mathrm{p}=0.57\end{array}$ \\
\hline
\end{tabular}

EPDS, Edinburgh Postnatal Depression Scale; MD, mean difference.

practised various techniques for an average total of 12.94 $(\mathrm{SD}=9.7)$ times per week. Women in the control group did not report antenatal practice of techniques, but some $(<5 \%)$ did report using techniques, such as breathing or visualization, during labour. No individual CM technique nor number of rehearsals in the antenatal period was associated with reduced likelihood of EDB use in the study group, indicating the overall effect of the programme.

To examine if there was any preference for therapies used during labour, we asked women in the study group $(n=88)$ what specific CM therapies they used during labour. On average, women used $3.94(\mathrm{SD}=1.4)$ techniques over the duration of their labour and in order of frequency used, BBs were used most frequently by $60.2 \%$ of women; visualisation was used by $55.7 \%$; acupressure by $46.6 \%$; yoga and massage, each done by $45.5 \%$ of women; and GBs were used by $35.2 \%$ of women during labour.

\section{DISCUSSION}

Main findings

The RCT demonstrated the effectiveness of the CTLB study, based on the She Births Antenatal Education Program and acupressure for labour protocol ${ }^{36}$ for firsttime mothers, by showing an absolute reduction of $45 \%$ and a relative reduction of $63 \%(\mathrm{RR}=0.37, \mathrm{p}<0.001)$ in epidural rate in the study group compared with controls. The study also showed increased rates of normal vaginal birth without surgical or mechanical assistance, and found reduced rates of augmentation in labour, length of second stage of labour, perineal trauma, CS and the need for resuscitation of the newborn. However, univariate results for secondary outcomes should be interpreted with caution as these are likely to be related to the primary outcomes of EDB, which has been shown to mediate the effect of these secondary outcomes. ${ }^{37} 38$ Additionally, where response rates for secondary analyses are low, the results should be interpreted with caution.

We note that women in the control group experienced a higher than average rate of EDB use, augmentation and instrumental vaginal birth, which is consistent with data showing higher rates of intervention for nulliparous women compared with multiparous women. ${ }^{10}$ The data for EDB use in this study are consistent with rates for women who are identified as being anxious. ${ }^{39} 40$ Further research is needed to identify if women who are anxious are more likely to participate in antenatal education programmes, and whether these women may benefit more from this type of intervention.

Current antenatal education has undergone a distinct shift towards normalising all births and preparing parents for parenthood. However, specific preparation for normal labour appears to have been de-emphasised in classes. ${ }^{41-43}$ Anecdotally, the majority of women attend routine antenatal education classes, but there is no current literature to provide accurate numbers. ${ }^{44} 45$ The results from the Cochrane Systematic Review suggest that while antenatal education aims to prepare women and partners for childbirth and early parenting, studies to date have shown a lack of high-quality evidence and a high variability of outcome measures. Therefore, the effects of antenatal education are still largely unknown. ${ }^{15}$ Studies exploring the use of antenatal education interventions, antenatal mindfulness training and self-hypnosis training have failed to demonstrate any reduction in the use of analgesia during labour and birth or on CS rates. ${ }^{15} 46-51$

Some commentators suggest that the impact of antenatal education in routine care may in fact be reinforcing medical management of labour and birth, and is therefore not addressing the rising rates of medical pain relief and the associated complications. ${ }^{7} 41435253$ In this study, we assessed if women from the control group used CM techniques, as a demonstration of cross-over. However, $<5 \%$ of women reported using these techniques during their labour, and this is unlikely to introduce any contamination to the results.

In this study, we emphasised the importance of reorienting the concept of normal birth using an antenatal education framework and a variety of evidence-based integrative $\mathrm{CM}$ techniques to help women manage pain in labour and birth. One of the recently voiced concerns of using alternative birth positions, such as yoga postures and upright positions, is the potential for increased risk of perineal trauma. ${ }^{54}$ The data from our research showed a statistically significant reduction in perineal trauma for women. Among those women who had vaginal births, $84.7 \%$ of the study group compared with $96.4 \%$ of the control group sustained some kind of perineal trauma during birth $(\mathrm{RR}=0.88, \mathrm{p}=0.02)$. Techniques were rehearsed in the antenatal period with some acupressure for induction techniques practiced lightly from 37 weeks as per the published literature. ${ }^{36}$ This is reported to work with the hormones that are naturally present in the woman's body, but do not artificially induce contractions. This is an important safety outcome, and there were no differences in gestational age at birth.

The study provides evidence that antenatal education integrating CM techniques is an effective and viable method of managing pain, decreasing medical interventions and increasing personal control for women. These 
clinically and statistically significant results are important in establishing an evidence base for the use and effectiveness of antenatal education programmes incorporating CM techniques for the management of pain during childbirth as an adjunct to parent education offered as usual care. This programme has the potential to provide a cost-effective method for antenatal education. A costing and economic analysis of this programme will be undertaken and reported elsewhere, providing a measure of relative benefit for outcomes saved. Reorienting antenatal education classes towards supporting normal birth, and providing techniques to help women manage pain is an important contribution for reducing interventions in labour and birth.

\section{Interpretation}

Our study helps to address the question of whether antenatal education using $\mathrm{CM}$ techniques are effective in reducing rates of EDB in first-time mothers. This primary outcome finding and other secondary findings, increased normal vaginal births and reduced augmentation, perineal trauma and CS, support some of the CM literature which show a reduction in rates of pharmacological pain relief, and some interventions during labour. ${ }^{22} 24-26$ These findings are in contrast to the parent education literature, hypnosis and psychoprophylaxis training literature for reduction of EDB during labour. ${ }^{15} 46495155-57$ The outcome of increase in positive attitude towards birth in the antenatal period and increased feelings of influence during labour and birth are supportive of the antenatal education literature. ${ }^{1516414855}$ The finding that no individual technique was associated with reduced rates of EDB highlights the concept that these techniques form a 'toolkit' of techniques and represent an overall holistic approach towards labour and birth. The combination of active birth techniques with relaxation techniques is unique to this programme.

The primary outcome measure of EDB was used for this study rather than pain scores which are frequently used in other CM studies. ${ }^{22}$ The objective measure of EDB has been identified as a mediating factor and is shown to influence labour interventions and mode of birth, which is described in the literature as the cascade of interventions. ${ }^{7} 810 \quad 3858$ The literature highlights the mechanism whereby an initial intervention during labour triggers subsequent interventions to manage the effects of the prior intervention. EDB has been shown to mediate this effect and is associated with outcomes such as augmentation during labour, instrumental vaginal birth and CS. ${ }^{37}$ This study demonstrates an impact on rates of EDB, as well as on rates of augmentation, perineal trauma and CS, and therefore may have an effect on the cascade of interventions. Therefore, caution is required when interpreting secondary outcome measures.

It remains important that methods used during labour are suitable for women's individual requirements and circumstances, and also account for conditions that may arise in the woman or infant during labour. ${ }^{20}$ This study demonstrates the capacity for a novel integrative antenatal education programme using $\mathrm{CM}$ techniques to reduce interventions in normal labour.

\section{Future research}

Policy initiatives supporting normal birth require novel solutions, and this study provides good evidence for such an initiative, including the potential for a revision of clinical practice in antenatal education. Future health services research should include translation of study outcomes into clinical practice, involving a priori costeffectiveness analysis, exploring key stakeholders' views about changing practice and undertaking a multicentred international study to assess the impact of the study in a broader context and beyond Australia. This article reports on the first implementation of this antenatal education programme, and evaluates feasibility of conduct. We are seeking to establish a larger trial in a broader national and international setting whereby issues of implementation and generalisability may be addressed. As a first stage, these results are promising and further investigation is warranted.

\section{CONCLUSION}

The rise in interventions rates in labour and birth need to be addressed as a matter of priority as outlined by reviews of maternity services ${ }^{3} 11$ and international reports. ${ }^{12}$ The high use of EDB for pain relief in labour has been identified as a contributing factor in rising rates of interventions, including CS. ${ }^{4-8}{ }^{20}$ This study highlights the effectiveness of a novel integrated antenatal education approach, incorporating evidence-based $\mathrm{CM}$ techniques to reduce rates of EDB and leading to a reduction in other interventions in labour and birth, including CS. This programme is novel in its approach and forms a unique toolkit for women and partners to use in their labour and birth.

The reorientation of antenatal education and the promotion of birth as a normal physiological event is critical if we are to reduce interventions in birth. This shift requires education and support to help women manage challenges faced during labour and birth. The results from this study demonstrate the potential effectiveness of the CT for labour and birth in providing an individualised, evidence-based, woman-centred, integrated approach to care that reduces medical interventions and morbidity in labour.

Twitter Follow Kate Levett at @LevettKate

Acknowledgements She Births provided background intellectual property, including concept, course materials and training. Five of the six CM techniques used formed part of the She Births programme (Nadine Richardson is founder of the She Births Antenatal Education Program e: nadine@shebirths.com p: +61 4124722 47). Dr Debra Betts provided the acupressure protocol for labour and birth. Neither was directly involved in this study. Paul Fahey provided all statistical support. The authors are grateful to 
all of the women, partners and midwives who participated in this trial, and to the midwifery and clerical staff at the two hospitals ' $\mathrm{H}$ ' and ' $\mathrm{N}$ ' who made a significant contribution to the conduct of the study, particularly Julia Wood and Hanni Witt.

Contributors KML, as the PhD student, was the main contributor to the development and design of the study, the conduct of the study, and drafting of the manuscript. CAS was the primary supervisor and HGD was the second supervisor, and each assisted with the research design, review of data and manuscript drafts. $A B$ is the third supervisor, and assisted with research design and review of manuscript.

Funding This article presents independent research, and the researcher is funded by an Australian Postgraduate Award, and a Postgraduate stipend from the Western Sydney University (WSU). Additional support in the form of RTS funds was given from the National Institute of Complementary Medicine (NICM) at WSU

Competing interests None declared.

Ethics approval The study was approved by the WSU ethics committee (H9579), Northern Sydney Local Health District (NSLHD) ethics committee (1111-476M, NEAF: HREC/11/H/268), and has site specific approval from the Western Sydney LHD (WSLDH) ethics committee (SSA/12/N/58), and was registered with the Australian New Zealand Clinical Trials Registry (ANZCTR) on 27 October.

Provenance and peer review Not commissioned; externally peer reviewed.

Data sharing statement Additional data may be available on request by emailing K.Levett@westernsydney.edu.au.

Open Access This is an Open Access article distributed in accordance with the Creative Commons Attribution Non Commercial (CC BY-NC 4.0) license, which permits others to distribute, remix, adapt, build upon this work noncommercially, and license their derivative works on different terms, provided the original work is properly cited and the use is non-commercial. See: http:// creativecommons.org/licenses/by-nc/4.0/

\section{REFERENCES}

1. Gibbons L, Belizán JM, Lauer JA, et al. The global numbers and costs of additionally needed and unnecessary caesarean sections performed per year: overuse as a barrier to universal coverage. World Health Rep 2010;30:1-31.

2. WHO. Caesarean sections should only be performed when medically necessary. Executive summary. In: WHO, ed. Geneva: WHO, 2015:8

3. Bryant R. Improving maternity services in Australia: the report of the maternity services review. In: Australia Co, ed. Canberra: Commonwealth of Australia, 2009.

4. Anim-Somuah M, Smyth RM, Jones L. Epidural versus non-epidural or no analgesia in labour. Cochrane Database Syst Rev 2011(12): CD000331.

5. King T. Epidural anesthesia in labor benefits versus risks. J Nurse Midwifery 1997;42:377-88.

6. Dahlen HG, Schmied V, Dennis CL, et al. Rates of obstetric intervention during birth and selected maternal and perinata outcomes for low risk women born in Australia compared to those born overseas. BMC Pregnancy Childbirth 2013;13:100.

7. Green JM, Baston HA. Have women become more willing to accept obstetric interventions and does this relate to mode of birth? Data from a prospective study. Birth 2007;34:6-13.

8. Roberts CL, Tracy S, Peat B. Rates for obstetric intervention among private and public patients in Australia: population based descriptive study. BMJ 2000;321:137-41.

9. Centre for Epidemiology and Evidence. New South Wales mothers and babies 2010. In: Evidence CfEa, ed. Sydney: NSW Ministry of Health, 2012

10. Dahlen HG, Tracy S, Tracy M, et al. Rates of obstetric intervention among low-risk women giving birth in private and public hospitals in NSW: a population-based descriptive study. BMJ Open 2012;2:pii: e001723.

11. NSW Department of Health. Maternity-towards normal birth in NSW. In: Health N, ed. Policy directive. Sydney: NSW Health, 2010.

12. Zwelling $\mathrm{E}$. The history of Lamaze continues: an interview with Elisabeth Bing. J Perinat Educ 2000;9:15-21.
13. Zwelling E. Down memory lane: recollections of Lamaze International's First 50 Years. J Perinat Educ 2010;19:11-16.

14. Svennson J, Barclay L, Cooke M. Effective antenatal education: strategies recommended by expectant and new parents. J Perinat Educ 2008;17:33-42.

15. Gagnon AJ, Sandall J. Individual or group antenatal education for childbirth or parenthood, or both. Cochrane Database Syst Rev 2007 (3):CD002869.

16. Jaddoe VW. Antenatal education programmes: do they work? Lancet 2009;374:863-4.

17. Smith CA, Collins CT, Cyna AM, et al. Complementary and alternative therapies for pain management in labour. Cochrane Database Syst Rev 2006(4):CD003521.

18. National Institute of Health. National center for complemenary and integrative health. In: $\mathrm{NIH}$, ed. $\mathrm{NCCIH}$. Bethesda, MA: NIH, National Center, 2015.

19. Steel A, Adams J, Sibbritt D, et al. The influence of complementary and alternative medicine use in pregnancy on labor pain management choices: results from a nationally representative sample of 1,835 women. J Altern Complement Med 2014;20: 87-97.

20. Jones L, Othman M, Dowswell T, et al. Pain management for women in labour: an overview of systematic reviews. Cochrane Database Syst Rev 2012;3:CD009234.

21. Craig $P$, Dieppe $P$, Macintyre $S$, et al. Developing and evaluating complex interventions: the new Medical Research Council guidance. BMJ 2008;337:a1655.

22. Levett KM, Smith CA, Dahlen HG, et al. Acupuncture and acupressure for pain management in labour and birth: a critical narrative review of current systematic review evidence. Complement Ther Med 2014;22:523-40.

23. Betts D. Acupressure techniques for use during childbirth and pregnancy. Childbirth solutions Online. (accessed 2015 2005).

24. Smith CA, Collins CT, Crowther CA, et al. Acupuncture or acupressure for pain management in labour. Cochrane Database Syst Rev 2011;(7):CD009232.

25. Smith CA, Levett KM, Collins CT, et al. Relaxation techniques for pain management in labour. Cochrane Database Syst Rev 2011; (12):CD009514

26. Smith CA, Levett KM, Collins CT, et al. Massage, reflexology and other manual methods for pain management in labour. Cochrane Database Syst Rev 2012;(2):CD009290.

27. Leap N, Dodwell M, Newburn M. Working with pain in labour. An overview of the evidence. New Digest 2010;49:22-6.

28. Field T. Yoga clinical research review. Complement Ther Clin Pract 2011;17:1-8.

29. Copstick SM, Taylor KE, Hayes R, et al. Partner support and the use of coping techniques in labour. J Psychosom Res 1986;30:497-503.

30. Leap N, Sandall J, Buckland S, et al. Journey to confidence: women's experiences of pain in labour and relational continuity of care. J Midwifery Womens Health 2010;55:234-42.

31. May C, Fletcher R. Preparing fathers for the transition to parenthood: Recommendations for the content of antenatal education. Midwifery 2013;29:474-8.

32. Cox JL, Holden JM, Sagovsky R. Detection of postnatal depression. Development of the 10-item Edinburgh Postnatal Depression Scale. Br J Psychiatry 1987;150:782-6.

33. Hodnett ED, Simmons-Tropea DA. The labour agentry scale: psychometric properties of an instrument measuring control during childbirth. Res Nurs Health 1987;10:301-10.

34. Li Z, Zeki R, Hilder L, et al. Australia's mothers and babies 2011. In: AlHW, ed. Perinatal statistics series no. 28. Cat. no. PER 59. Canberra: AlHW National Perinatal Epidemiology and Statistics Unit, 2013.

35. Fewtrell MS, Kennedy K, Singhal A, et al. How much loss to follow-up is acceptable in long-term randomised trials and prospective studies? Arch Dis Child 2008;93:458-61.

36. Betts D. The use of acupuncture as a routine pre-birth treatment J Chin Med Hove 2004:5-8.

37. Rossignol M, Chaillet N, Boughrassa $\mathrm{F}$, et al. Interrelations between four antepartum obstetric interventions and cesarean delivery in women at low risk: a systematic review and modeling of the cascade of interventions. Birth 2014;41:70-8.

38. Tracy SK, Tracy MB. Costing the cascade: estimating the cost of increased obstetric intervention in childbirth using population data. BJOG 2003;110:717-24.

39. Rouhe $\mathrm{H}$, Salmela-Aro K, Toivanen $\mathrm{R}$, et al. Obstetric outcome after intervention for severe fear of childbirth in nulliparous womenrandomised trial. BJOG 2013;120:75-84. 
40. Sjögren B, Thomassen P. Obstetric outcome in 100 women with severe anxiety over childbirth. Acta Obstet Gynecol Scand 1997;76:948-52.

41. Ferguson S, Davis D, Browne J. Does antenatal education affect labour and birth? A structured review of the literature. Women Birth 2013;26:e5-8.

42. Murphy Tighe S. An exploration of the attitudes of attenders and nonattenders towards antenatal education. Midwifery 2010;26:294-303.

43. Walker DS, Visger JM, Rossie D. Contemporary childbirth education models. J Midwifery Womens Health 2009;54:469-76.

44. Lumley J, Brown S. Attenders and nonattenders at childbirth education classes in Australia: how do they and their births differ? Birth 1993;20:123-30.

45. Bennett C, Shearman R. Maternity services in New South Waleschildbirth moves toward the 21st century. Med J Aust 1989;150:673-6.

46. Downe S, Finlayson K, Melvin C, et al. Self-hypnosis for intrapartum pain management in pregnant nulliparous women: a randomised controlled trial of clinical effectiveness. BJOG 2015;122:1226-34.

47. Bergström $\mathrm{M}$, Kieler $\mathrm{H}$, Waldenström $\mathrm{U}$. Natural childbirth vs antenatal education. Midwives 2009;12:21.

48. Byrne J, Hauck Y, Fisher C, et al. Effectiveness of a mindfulness-based childbirth education pilot study on maternal self-efficacy and fear of childbirth. J Midwifery Womens Health 2014;59:192-7.

49. Cyna AM, Crowther CA, Robinson JS, et al. Hypnosis antenatal training for childbirth: a randomised controlled trial. BJOG 2013;120:1248-59; discussion 1256-7.
50. Koehn ML. Childbirth education outcomes: an integrative review of the literature. J Perinat Educ 2002;11:10-19.

51. Madden K, Middleton P, Cyna AM, et al. Hypnosis for pain management during labour and childbirth. Cochrane Database Syst Rev 2016;(5):CD009356

52. Lothian JA. Listening to mothers II: knowledge, decision-making, and attendance at childbirth education classes. J Perinat Educ 2007;16:62-7.

53. Lothian JA. Childbirth education at the crossroads. J Perinat Educ 2008;17:45-9.

54. Gupta JK, Hofmeyr GJ. Position for women during second stage of labour. Cochrane Database Syst Rev 2004;(1):CD002006.

55. Bergström M, Kieler $\mathrm{H}$, Waldenström $\mathrm{U}$. Effects of natural childbirth preparation versus standard antenatal education on epidural rates, experience of childbirth and parental stress in mothers and fathers: a randomised controlled multicentre trial. BJOG 2009;116:1167-76.

56. Bergström $\mathrm{M}$, Kieler $\mathrm{H}$, Waldenström $\mathrm{U}$. A randomised controlled multicentre trial of women's and men's satisfaction with two models of antenatal education. Midwifery 2011;27: e195-200.

57. Cyna AM, McAuliffe GL, Andrew MI. Hypnosis for pain relief in labour and childbirth: a systematic review. Br J Anaesth 2004;93:505-11.

58. Tracy SK, Sullivan E, Wang YA, et al. Birth outcomes associated with interventions in labour amongst low risk women: a population-based study. Women Birth 2007;20:41-8. 


\section{Correction: Complementary therapies for labour and birth study: a randomised controlled trial of antenatal integrative medicine for pain management in labour}

Levett KM, Smith CA, Bensoussan A, et al. Complementary therapies for labour and birth study: a randomised controlled trial of antenatal integrative medicine for pain management and labour. BMJ Open 2016;6:e010691 doi:10.1136/bmjopen2015-010691.

There are several amendments to this article:

Reference 23 should be Betts D. Acupressure techniques for use during childbirth and pregnancy. http://acupuncture.rhizome.net.nz (accessed 2015 2005).

The sentence: Acupressure, ${ }^{22} 24$ which uses six main points for use during labour selected from a previously published protocol. ${ }^{23}$ These focus on hormone release for labour progression, augmentation of contractions, pain relief, nausea and positioning of baby.

Should read: Acupressure, ${ }^{22} 24$ which uses six main points for use during labour selected from a previously published protocol. ${ }^{23}$ The participants were given DVDs of the acupressure protocol ${ }^{23}$ to take home for practice. These focus on hormone release for labour progression, augmentation of contractions, pain relief, nausea and positioning of baby.

The sentence: The LAS contains 29 questions with a seven-point Likert scale ranging from ' $1=$ almost always', to ' $7=$ =rarely'. Therefore, scores could theoretically range from 29, indicating the highest control possible, to a high score of 203 indicating the lowest agency possible.

Should read: The LAS contains 29 questions with a seven-point Likert scale ranging from ' $1=$ almost always', to ' $7=$ =rarely'. Therefore, scores could theoretically range from 29, indicating the lowest control possible, to a high score of 203 indicating the highest agency possible.

The acknowledgements have been corrected to include: Dr Debra Betts provided the acupressure protocol for labour and birth and can be accessed at this address: https://acupuncture.rhizome.net.nz/). Dr Debra Betts (debra.betts@rhizome.net.nz) and Tom Kennedy (tzkennedy@hotmail.com) provided the DVD for the study participants. None were directly involved in this study.

Reference 1 in the supplementary data has been corrected to:

Reference 1: Betts D. Acupressure techniques for use during childbirth and pregnancy. http://acupuncture.rhizome.net.nz (accessed 2015 2005).

Open Access This is an Open Access article distributed in accordance with the Creative Commons Attribution Non Commercial (CC BY-NC 4.0) license, which permits others to distribute, remix, adapt, build upon this work noncommercially, and license their derivative works on different terms, provided the original work is properly cited and the use is non-commercial. See: http://creativecommons.org/licenses/by-nc/4.0/

BMJ Open 2016;6:e010691corr1. doi:10.1136/bmjopen-2015-010691corr1 